\title{
Mucous membrane pemphigoid as a cause of acute dysphagia - an endoscopic study
}

\author{
Karolina Sawicka ${ }^{1}$, Jerzy Baraniak ${ }^{2}$, Małgorzata Michalska-Jakubus ${ }^{1}$, Małgorzata Przyszlak-Szabała ${ }^{1}$, \\ Aldona Pietrzak¹, Jerzy Mosiewicz², Jadwiga Sierocińska-Sawa³, Grażyna Chodorowska ${ }^{1}$, Dorota Krasowska ${ }^{1}$ \\ 1Department of Dermatology, Venereology, and Paediatric Dermatology, University Hospital No. 1, Lublin, Poland \\ ${ }^{2}$ Department of Internal Medicine, University Hospital No. 1, Lublin, Poland \\ ${ }^{3}$ Pathomorphology Laboratory, University Hospital No. 1, Lublin, Poland
}

Prz Gastroenterol 2015; 10 (4): 247-249

DOI: $10.5114 /$ pg.2015.49475

Address for correspondence: Karolina Sawicka MD, Department of Dermatology, Venereology, and Paediatric Dermatology, University Hospital No. 1, 1 Radziwiłłowska St, 20-080 Lublin, Poland, phone/fax: +48 8153236 47, e-mail: Karolina.kowalczyk4@gmail.com

Mucous membrane pemphigoid (MMP, formerly cicatricial pemphigoid) is a chronic autoimmune, subepithelial blistering disease usually of middle aged or elderly patients, more common in women. The prevalence is uncertain and the aetiology is still unknown. Target antigens are epithelial basement membrane proteins BPAG-1, BPAG-2, laminin-331, and laminin-332 [1]. Oral and conjunctival membranes are most frequently affected. Oesophageal involvement is rare but may be complicated with persistent dysphagia [2].

Herein, we present a patient with MMP with acute dysphagia evaluated by upper gastrointestinal (GIT) endoscopy. This study is based on a case report of a 75-year-old patient with MMP. Clinical and endoscopic examinations as well as direct immunofluorescence (DIF) of conjunctival biopsy were used as methods in this study.

A 75-year-old woman was referred to the Dermatology Clinic in November 2011 due to painful erosions in the oral cavity and dysphagia. Her past medical history was significant for dry-eye sensations with visual disturbances diagnosed as ocular MMP in 2007 confirmed by DIF (IgA+ deposits) and treated with dapsone. On physical examination there were erosions and haemorrhagic blisters on the hard palate (Figure $1 \mathrm{~A}$ ). Inspection of the eyes revealed bilateral conjunctival hyperaemia and fibrous adhesions between bulbar and palpebral conjunctiva (symblepharon) (Figure 1 B). No laboratory changes were found, and autoantibodies in the blood (indirect immunofluorescence) were negative. An endoscopic examination of the upper GIT was performed and revealed: 1) white fibrin with or without flat erosions on the mucosa of cricopharynx, along the oesophagus, and on the lining of the stomach body and pre- pyloric region; 2) two flat, linear erosions, about $10 \mathrm{~mm}$ long on the lower third of the oesophagus; 3 ) hiatal hernia with line " $Z$ " $4 \mathrm{~cm}$ above the diaphragm; and 4) spastic but passable pylorus and erythematous mucosa of duodenal bulb (Figure $1 \mathrm{C}$ ). An endoscopic biopsy from the oesophagus showed separation between epithelium and mucosa (Figure 1 D). Given the final diagnosis of MMP with prominent conjunctival and oro-oesophageal involvement, the patient was started on methylprednisolone $16 / 8 \mathrm{mg}$ per day, which resulted in resolution of oral lesions and dysphagia. She remains stable on maintenance therapy with low-dose methylprednisolone. At 18-months' follow-up the patient remains under control.

Most patients (89-100\%) manifest with oral involvement [1]. The conjunctival mucosa is the second most frequently involved site (66\%) [1]. Mucous membrane pemphigoid typically begins with blisters or erosions on mucous membranes or skin. Symptoms of MPP evolve over several weeks with subsequent exacerbation and remission periods. The primary lesion is a serous or haemorrhagic blister of variable size that ruptures in 24 to $48 \mathrm{~h}$ producing erosion or ulceration that heals slowly and usually tends toward scarring [1]. Mucous membrane pemphigoid is characterised by deposits of IgG, IgA, or C3 complement along the basement membrane zone. Antigens of epithelium are regarded as targets for autoantibodies. These autoantigens are basically BPAG-1, BPAg-2, laminin-331, and laminin-332, which belong to epithelial proteins [3]. Oral lesions present as painful erosions on buccal mucosa and the palate or as desquamative gingivitis, which is very typical of MMP [4-6]. When dysphagia is observed, it is usually secondary to the involvement of hypopharynx. Oesophageal 

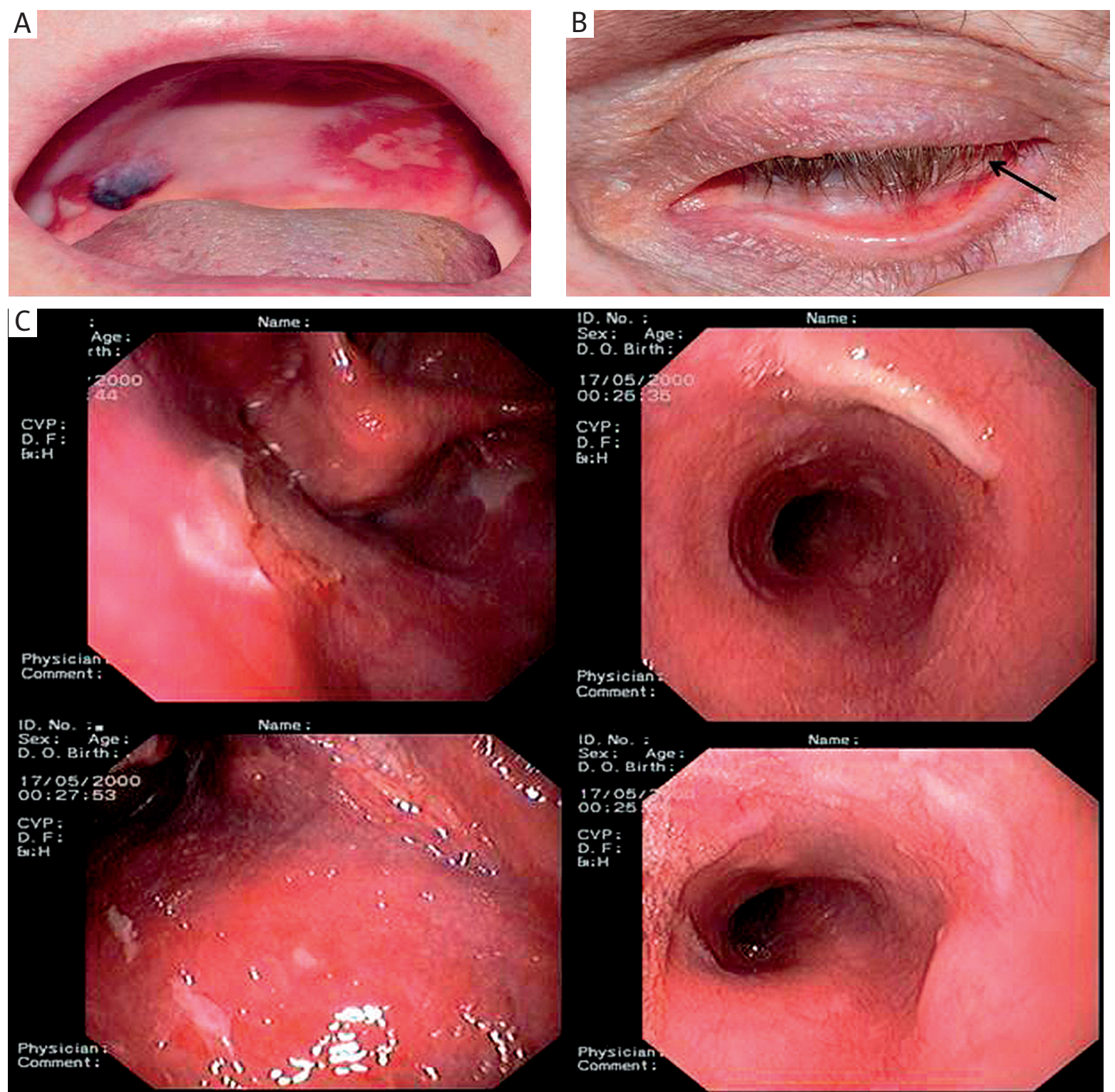

Physician
Comment:
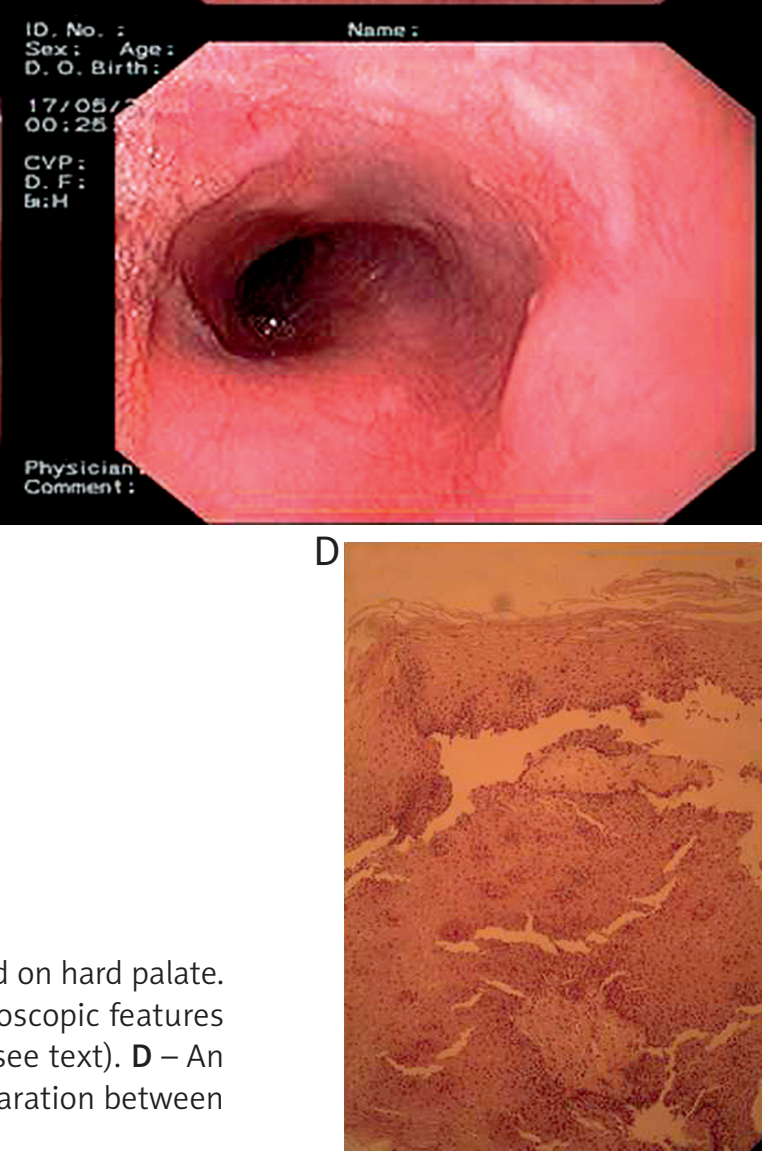

Figure 1. A - Haemorrhagic blister and erosion localised on hard palate. B - Eye involvement - symblepharon (arrow). C - Endoscopic features of oesophageal involvement in a 75-year-old patient (see text). D - An endoscopic biopsy from oesophagus $(H+E, 40 x)$ - separation between epithelium and mucosa 
affection is present in up to $15 \%$ of cases and rarely as first or isolated presentation [4]. Endoscopic features of oesophageal involvement vary at different stages of the disease and include longitudinal folding of the oesophageal mucosa and diffuse nonspecific inflammatory changes (mucosal oedema, areas of inconstant narrowing and spasm, erosions, superficial ulcerations), which heal with scarring, leading to the formation of adhesions, transverse and circumferential webs, and various sizes of strictures. Oesophageal involvement may be completely asymptomatic or complicated with dysphagia and even life-threatening bleeding from upper GIT $[7,8]$. It may manifest as various types of lesions including esophagitis dissecans superficialis (EDS), as a most severe but rare form. Typical features of EDS are vertical fissures and sloughing of whitish superficial epithelium, resulting in dysphagia, odynophagia, and heartburn, but haematemesis or vomiting oesophageal casts occurs rarely [7]. Adhesions appear as healing progresses and scar formation develops. Strictures result from recurrent episodes of disease may be single or multiple and of variable length, sometimes requiring endoscopic dilatation or surgery [7]. Bullae are rarely seen because endoscopy is usually performed at a late stage when scarring has already occurred [4]. Upper endoscopy is not a routine method in the investigation of patients with MMP. It should be indicated in patients with odynophagia, epigastric pain, or bleeding of the gastrointestinal tract [8]. The course of MMP is progressive. The disease seldom goes into complete remission spontaneously, and the aim of therapy is to prevent progression. Treatment strategies depend on the extent and severity of the disease [4]. Most patients require systemic therapy, and corticosteroids (prednisone or methylprednisolone) are the drugs of choice. Immunosuppressants and Dapsone have also been used with success $[1,5]$. Some studies show promising results with the use of mycophenolate mofetil or etanercept $[9,10]$.

The presented case shows the importance of endoscopic diagnostics in acute dysphagia, regardless of the underlying disease. It is also worth emphasising that patients with MMP must be followed up because the disease may relapse, often in a different localisation.

\section{Conflict of interest}

The authors declare no conflict of interest.

\section{References}

1. Chan LS. Ocular and oral mucous membrane pemphigoid (cicatricial pemphigoid). Clin Dermatol 2012; 30: 34-7.

2. Bruch-Gerhaz D, Hertl M, Ruzicka T. Mucous membrane pemphigoid: clinical aspects, immunopathological features and therapy. Eur J Dermatol 2007; 17: 191-200.
3. Chan L. Mucous membrane pemphigoid. Clin Dermatol 2001; 19: 703-11.

4. Kwaśniewicz D, Białynicki-Birula R, Zaleska-Kręcicka M, et al. Cicatricial pemphigoid - case report. Otorynolaryngologia 2011; 10: 134-7.

5. Chan L, Ahmed R, Anhalt G, et al. The first international consensus on mucous membrane pemphigoid. Arch Dermatol 2002; 138: 370-9.

6. Petruzzi M. Mucous membrane pemphigoid affecting the oral cavity: short review on etiopathogenesis, diagnosis and treatment. Immunopharmacol Immunotoxicol 2012; 34: 363-7.

7. Hokama A, Yamamoto Y, Taira K, et al. Esophagitis dissecans superficialis and autoimmune bullous dermatoses: a review. World J Gastrointest Endosc 2010; 2: 252-6.

8. Eng TY, Hogan WJ, Jordan RE. Esophageal involvement in bullous pemphigoid - a possible cause of gastrointestinal hemorrhage. Br J Dermatol 1978; 99: 207-10.

9. Megahed M, Schmiedeberg S, Becker J, et al. Treatment of cicatricial pemphiogid with mycophenolate mofetil as a steroid-sparing agent. J Am Acad Dermatol 2001; 45: 256-9.

10. Canizares MJ, Smith DI, Conners MS, et al. Successful treatment of mucous membrane pemphigoid with etanercept in three patients. Arch Dermatol 2006; 142: 1457-61.

Received: 29.10.2014

Accepted: 7.12 .2014 